\title{
Near-field light focusing by wavelength-sized dielectric spheroids for photovoltaic applications
}

\author{
Manuel J. Mendes*, Ignacio Tobías, Antonio Martí and Antonio Luque \\ Instituto de Energía Solar, E.T.S.I. Telecomunicación, Universidad Politécnica de Madrid, Ciudad \\ Universitaria, Avda. Complutense 30, 28040 Madrid, Spain. \\ *Corresponding author: manuel.mendes@ies-def.upm.es
}

\begin{abstract}
We explore the near-field concentration properties of dielectric spheroidal scatterers with sizes close to the wavelength, using an analytical separation-of-variables method. Such particles act as mesoscopic lenses whose physical parameters are optimized here for maximum scattered light enhancement in photovoltaic applications.

OCIS codes: (290.5850) Scattering, particles; (260.2110) Electromagnetic optics; (040.5350) Photovoltaic.
\end{abstract}

\section{Introduction}

There has been a growing interest in near-field optics [1], stemmed by recent applications that can benefit from the enhanced local fields in the vicinity of scattering particles: resonance spectroscopy imaging (e.g. luminescence and Raman enhancement), localized sensing methods (e.g. back-scattering amplification), nanoscale processing of materials (e.g. photo-lithography), optical data storage, among others. However, the authors are primarily interested in developing near-field structures for light trapping in photovoltaic devices (e.g. quantum-dot solar cells [2]).

Near-field effects become important when the size of the scattering object is smaller or on the order of the incident illuminating wavelength $(\lambda)$; therefore outside the macroscopic regime of geometrical optics (GO). This work is focused on scatterers with sizes on the order of $\lambda$ - the mesoscopic regime. This regime is still rather unexplored since the analytical solution of electromagnetic (EM) scattering by mesoscopic particles requires the detailed calculation of the full set of Maxwell's equations [1].

Most of the analytical studies published so far use Mie theory [3, 4], valid for any particle size but restricted to spherical shapes. Such studies demonstrated that dielectric mesoscopic particles (DMPs) could produce pronounced electric fields intensities close to their shadow-side surface. They act as near-field lenses concentrating the light in a jet-like "focal region" located along the incidence axis, which was corroborated experimentally by direct imaging [5]. However, in most cases of practical interest scatterers are non-spherical and can be better approximated by a spheroidal shape. Therefore, here a spheroidal coordinate separation-of-variables solution [6, 7] is used to explore the near-field light focusing properties of spheroidal DMPs with arbitrary size, aspect ratio and complex refractive index. The results reveal important additional possibilities relative to the particular case of spheres.

DMPs can be used as "mesoscopic lenses" in light management procedures applied to solar cells [8]. They are shown to exhibit remarkable light concentration properties that surpass the limitations of macroscopic GO. By optimally tuning the spheroid parameters (size, aspect ratio and refractive index) one can shape the region of constructive interference of the scattered light in order to produce spots of strong electric intensity (above 100 times the incident intensity) or wide focal regions that can extend up to several wavelengths in front of the particle. The focused light forward scattered by DMPs can improve the absorption in a nearby photovoltaic (PV) medium; therefore allowing a reduction in the amount of expensive PV material, an improvement in the conditions for charge carrier collection and an increase in the efficiency by virtue of the concentrated energy density.

\section{Separation of Variables method and Optimization algorithm}

We consider the scattering of a plane-wave by a spheroidal particle whose axis of revolution is oriented along the incident wave direction $\left(\mathbf{K}_{\mathbf{0}}\right)$. Both the particle material and the surrounding medium are homogeneous and isotropic. The solution is obtained by expanding the scattered, internal and incident fields in terms of an infinite sum of spheroidal vector wave functions whose coefficients are determined from the boundary conditions of continuity at the spheroid-medium interface. The method is described in detail in [6,7]. The coordinate system is depicted in Fig. 1a). The illuminating wave propagates along the negative $z$ direction with the electric field $\mathbf{E}_{\mathbf{0}}$ parallel to $y$. The length unit used in this work is $\lambda$, and the electric field unit is $\mathrm{E}_{0}$. 


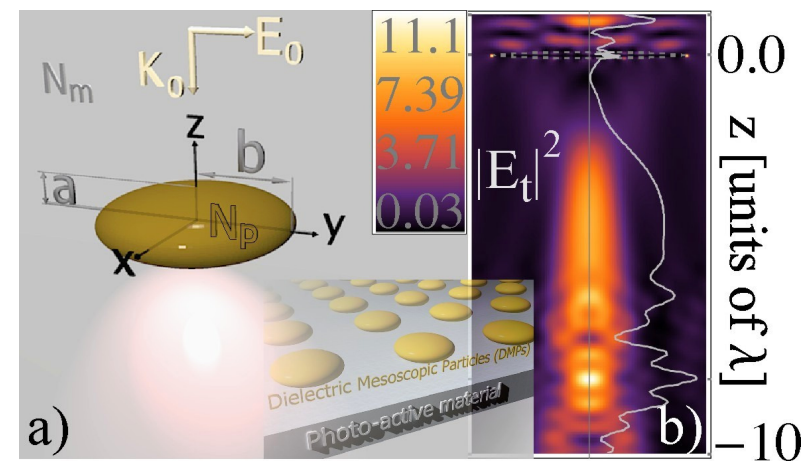

Fig. 1: a) Coordinate system with origin at the spheroid center. The particle refractive index $\left(N_{p}\right)$ is higher than that of the ambient medium $\left(N_{m}\right)$. An array of such particles embedded or placed on the surface of a PV material could enhance its light absorption, as sketched in the bottom inset. b) Total electric field intensity $\left|\mathbf{E}_{\mathbf{t}}\right|^{2}$ distribution produced by an elongated oblate spheroid (outlined by the dashed line) with semiaxes $b=2.271 \lambda$ and $a=0.079 \lambda$ and $N_{r}=N_{p} / N_{m}=4+0.01$ i. The particle parameters were optimized to produce a high integral of the near-field intensity over a distance of $10 \lambda$ in front of the particle. The gray curve on the right side corresponds to $\left|\mathbf{E}_{\mathbf{t}}\right|^{2}$ on the $z$ axis.

The code used in this work was extensively checked with known analytic and numerical solutions [7]. Here further comparisons with the electrostatic approximation (EA) and Mie theory are presented in Fig. 2. The physical parameters involved are the complex relative refractive index $\left(N_{r}=n_{r}+\mathrm{i} k_{r}\right)$, the spheroid aspect ratio $(b / a)$ and the spheroid size given by the volume-equivalent-sphere radius $\left(R_{e q}\right)$. The absorptive nature of scatterers and the high confinement of their near-field substantially limit the parameter space where DMPs can provide exceptional improvements to the properties of the surrounding receiving materials (emitters or absorbers depending on the application). Therefore, a theoretical study involving a computational optimization is crucial prior to any practical implementation. In this work, a Nelder and Mead optimization algorithm was coupled to the described analytical method to search for the set of DMP parameters that provide the highest possible forward scattered field intensities along a certain region of the external medium. Fig. 1b) presents an example of a field distribution given by an optimally designed DMP that originates a broad focal region extending up to a distance of $10 \lambda$ below the particle. A small imaginary part $\left(k_{r}=0.01\right)$ is considered in $N_{r}$ for the results to meet conditions attainable in practice.

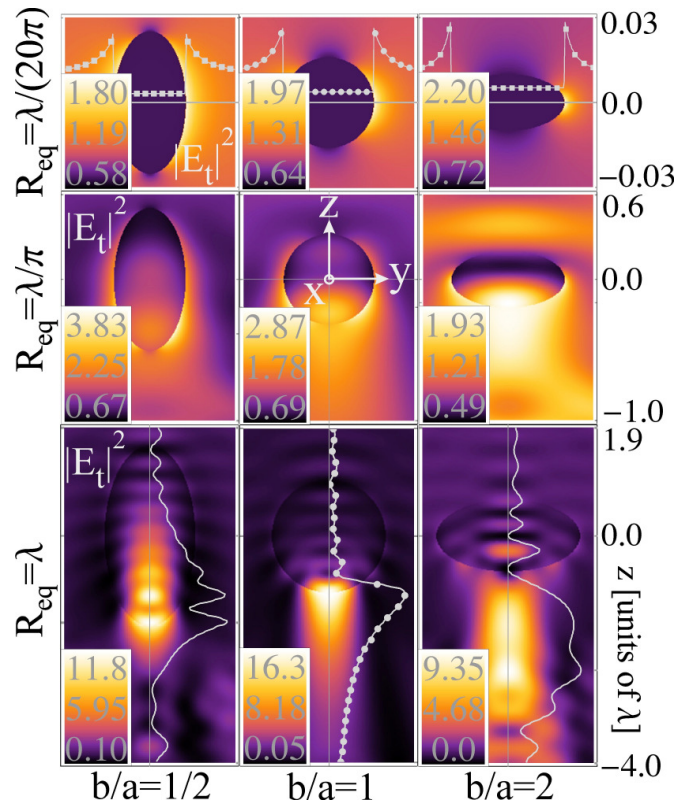

Fig. 2: Total electric intensity distribution produced by spheroids with distinct sizes $\left(R_{e q}\right)$ and shapes $(b / a) . N_{r}=1.33$ for all particles. In the top images the values of $\left|\mathbf{E}_{\mathbf{t}}\right|^{2}$ along the horizontal $y$ axis (gray lines) are compared with those obtained with EA (square dots). The gray lines on the bottom plots correspond to $\left|\mathbf{E}_{t}\right|^{2}$ on the $z$ axis. In the central plot for spheres $(b / a=1)$ the curve is compared with Mie theory (circular dots).

\section{Scattering size regimes}

The way in which the scatterer geometry influences the field around it changes considerably according to the size of the particle $\left(R_{e q}\right)$ relative to $\lambda$, as shown in the field distributions of Fig 2 . Optically small particles $\left(\operatorname{see} R_{e q}=\lambda / 20 \pi\right)$ 
act as electric dipoles (EA regime) producing an external peak field intensity on the $\mathbf{E}_{\mathbf{0}}$ axis (at $y= \pm b$ ). As the scatterer size increases and approaches $\lambda$ ( $\operatorname{see} R_{e q}=\lambda / \pi$ ) higher order modes (quadrupolar, octopolar, etc.) are excited. The bigger the particle the more modes come into play, causing the appearance of additional features in the field patterns that are highly dependent on the particle geometry and material. The peak field intensity moves from the $\mathbf{E}_{\mathbf{0}}$ axis to the forward direction $\left(\mathbf{K}_{\mathbf{0}}\right)$ as the size increases [7]. Particles with sizes close to or above $\lambda$ (see $R_{e q}=\lambda$ ) produce transverse polarized scattered fields focused along its axis of revolution $(z)$. Such scatterers act as optical cavities and its near-field distribution is a standing wave pattern in which the electric energy is removed from the regions of destructive interference and concentrated in those of constructive interference. A smaller volume of constructive interference implies a brighter focus since there is a higher density of electric energy. This resembles the focusing effect of a macroscopic biconvex lens. However, the characteristics of such focal spots are quite distinct from those predicted by GO [3, 4, 9]. The conventional GO focusing gives diffraction-limited focal spots. With DMPs the focal spots can have sub-wavelength dimensions, surpassing the diffraction limit.

\section{Maximum forward light focusing}

The focal region can be maximally confined in the medium outside the DMP if the spot of constructive interference occurs at the bottom surface of the particle on the $\mathbf{K}_{\mathbf{0}}$ axis (at $z=-a$ ) [3]. In that case, the focus can be localized in a sub-wavelength volume with maximum field strength. As observed in the bottom plots of Fig. 2, increasing the particle aspect ratio $b / a$ moves the region of highest field magnitude away from the particle, spreading the focus. Increasing the real part of the refractive index $\left(n_{r}\right)$ has the opposite effect (see Fig. 3a) and b)), and also enhances the interference caused by the DMP allowing the achievement of higher field intensities. An optimal adjust of $n_{r}$ and $b / a$ can therefore place the focus right at the $z=-a$ spot and allow extremely intense external fields of more than two orders of magnitude higher than the incident field intensity, as shown in Fig. 3c).
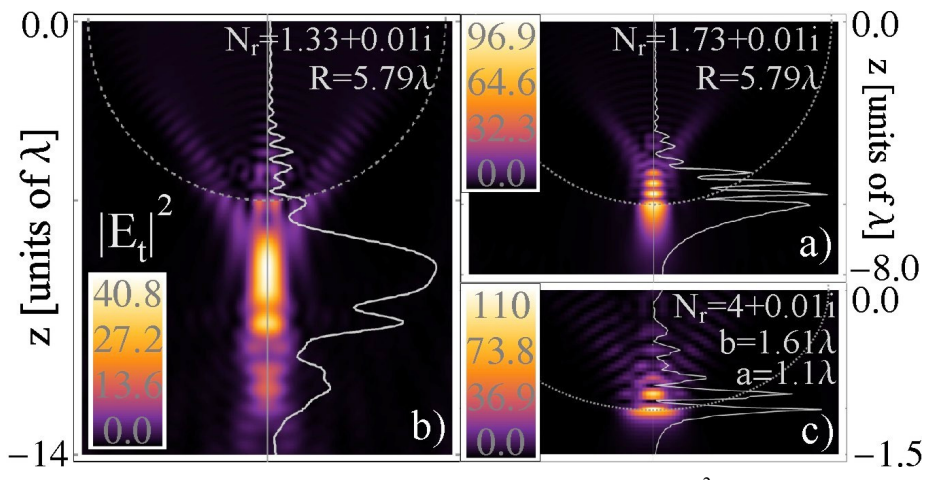

Fig. 3: a) Result of the optimization of the sphere parameters $R$ and $n_{r}$ that maximize $\left|\mathbf{E}_{\mathrm{t}}\right|^{2}$ outside a spherical particle (outlined by the dashed line) with $k_{r}=0.01$ i. b) Field distribution produced by a sphere with the same parameters as in a) but with smaller $n_{r}=1.33$, shown for comparison $\mathbf{c})$ Spheroid whose parameters $\left(R_{e q}, b / a\right.$ and $\left.n_{r}\right)$ were optimized to produce the highest $\left|\mathbf{E}_{t}\right|^{2}$ at a spot in the external medium.

\section{Acknowledgements}

This work was supported by the projects IBPOWER (Grant No. 211640), NUMANCIA-2 (Grant No. S2009/ENE1477) and DENQUIBAND (Grant No. PLE2009-0045). Manuel J. Mendes also acknowledges "Universidad Politécnica de Madrid" for the scholarship Beca de Doctorado Homologada.

\section{References}

[1] C. Girard and A. Dereux, "Near-field optics theories," Reports on Progress in Physics 59, 657-699 (1996).

[2] M. J. Mendes, A. Luque, I. Tobias, and A. Marti, "Plasmonic light enhancement in the near-field of metallic nanospheroids for application in intermediate band solar cells," Applied Physics Letters 95, 071105 (2009).

[3] S. Lecler, Y. Takakura, and P. Meyrueis, "Properties of a three-dimensional photonic jet," Optics Letters 30, 2641-2643 (2005).

[4] A. Devilez, N. Bonod, and B. Stout, "Near field dielectric microlenses," in Optical Modelling and Design, F. Wyrowski, J. T. Sheridan, J. Tervo, and Y. Meuret, eds. (Spie-Int Soc Optical Engineering, Bellingham, 2010).

[5] P. Ferrand, J. Wenger, A. Devilez, M. Pianta, B. Stout, N. Bonod, E. Popov, and H. Rigneault, "Direct imaging of photonic nanojets," Optics Express 16, 6930-6940 (2008).

[6] L.-W. Li, X.-K. Kang, and M.-S. Leong, Spheroidal Wave Functions in Electromagnetic Theory (John Wiley \& Sons, Inc., 2002).

[7] M. J. Mendes, I. Tobías, A. Martí, and A. Luque, "Near-field scattering by dielectric spheroidal particles with sizes on the order of the illuminating wavelength," J. Opt. Soc. Am. B 27, 1221-1231 (2010).

[8] J. Grandidier, D. M. Callahan, J. N. Munday, and H. A. Atwater, "Thin-Film Solar Cells: Light Absorption Enhancement in Thin-Film Solar Cells Using Whispering Gallery Modes in Dielectric Nanospheres (Adv. Mater. 10/2011)," Advanced Materials 23, 1171-1171 (2011).

[9] A. V. Itagi and W. A. Challener, "Optics of photonic nanojets," J. Opt. Soc. Am. A 22, 2847-2858 (2005). 